\title{
Physical Diagnosis of Pain: an Atlas of Signs and Symptoms - 3rd Edition
}

\author{
Steven D. Waldman. Elsevier, St Louis, Missouri, 2016, \$250.00, hardcover, 400 pages. \\ ISBN 978-0-323-37748-5
}

Yawar J. Qadri, MD, PhD • Lance A. Roy, MD

Received: 27 April 2016/Accepted: 9 May 2016/Published online: 12 May 2016

(c) Canadian Anesthesiologists' Society 2016

With the increased use of radiographic and sonographic examinations in clinical practice, the art of the physical examination is receiving less emphasis during medical training. Unfortunately, inadequate understanding of basic physical examination skills can lead to unnecessary interventions, procedures that are not indicated, and untreated or misdiagnosed pathology. These errors occur because it is the findings uncovered during the physical examination and provocative tests that are often critical to determining the source of a patient's pain. Perhaps with the renewed interest and focus on managing costs under the current health care delivery models, physical examination skills will be considered more essential as unneeded diagnostic tests are denied by payers.

With these concerns in mind, Physical Diagnosis of Pain: an Atlas of Signs and Symptoms provides an excellent tool for clinicians attempting to diagnose the anatomic pathology(ies) responsible for the patient's complaints. This updated third edition by Dr. Steven Waldman is an excellent reference for honing one's physical examination techniques and provocative maneuvers that help delineate and differentiate pain generators. Structured in a manner similar to that used for the classic 1976 textbook Physical Examination of the Spine and Extremities by Stanley Hoppenfeld, it maintains the template of previous editions, with the text separated into 11 anatomic areas beginning with the cervical spine and continuing through the ankle and foot.

Each section begins with an initial review of the basic anatomy of that area, with radiographic and

Y. J. Qadri, MD, PhD · L. A. Roy, MD ( $\varangle)$

Duke University Health System, Durham, NC, USA

e-mail: lance.roy@duke.edu ultrasonographic images provided where applicable. The images are well annotated and are easy to read and understand. After this brief anatomic review, Dr. Waldman provides clinically useful knowledge on the pathologies and painful conditions generated by dysfunction in that area, followed by relevant detailed descriptions, pictographs, and videos describing techniques used to identify the problem. Tables are provided to guide the practitioner quickly through the causes of, and differential diagnosis for, pain in each area.

Importantly for a reference book, each section is designed for rapid entry while providing adequate coverage of the topic. Each topic contains all of the relevant background information required for easy comprehension. For example, the adduction stress test for acromioclavicular joint dysfunction is concisely described, providing a rapid review, while also having the relevant radiographic and ultrasonographic images easily available within the same section.

Many sections and topics also have hyperlinks to videos that are made available with this edition as an online companion through Elsevier's Expert Consult service. These videos provide concise, rapid reinforcement of the text description, giving readers a quick visual impression of how to perform some of the more complex or less wellknown physical techniques. The text complements the videos well, without significantly relying on them. Indeed, many learners may prefer this easily accessible online video approach to a traditional text-based approach. Its inclusion is one of the main distinguishing factors that recommend the new edition.

Only a few minor weaknesses of the book are apparent. One is the paucity of discussion regarding the sensitivity or specificity of the various physical techniques, although this 
is perhaps indicative of a broader failing within the pain medicine community regarding this issue. The wide breadth of coverage also necessitates less detail regarding rarer pain pathologies. In addition, the book omits coverage of pain states involving the head or any central neuronal process.

In summary, Physical Diagnosis of Pain: an Atlas of Signs and Symptoms is an excellent stand-alone reference that would be helpful to providers seeking guidance on rapidly and effectively diagnosing pathology based on physical techniques, rather than relying on imaging alone.
It also is useful for those who need a quick refresher on physical examination techniques for a pain site they rarely examine or about which they need further clarity. We recommend this book to any provider who commonly evaluates and treats pain problems.

Conflicts of interest None declared.

Editorial responsibility This submission was handled by Dr. Hilary P. Grocott, Editor-in-Chief, Canadian Journal of Anesthesia. 